\title{
Association between leptin, body composition, sex and knee cartilage morphology in older adults: the Tasmanian older adult cohort (TASOAC) study
}

C Ding, V Parameswaran, F Cicuttini, J Burgess, G Zhai, S Quinn and G Jones

Ann Rheum Dis 2008;67;1256-1261; originally published online 3 Jan 2008; doi:10.1136/ard.2007.082651

Updated information and services can be found at:

http://ard.bmj.com/cgi/content/full/67/9/1256

References

Rapid responses

Email alerting service

\section{These include:}

This article cites 28 articles, 5 of which can be accessed free at: http://ard.bmj.com/cgi/content/full/67/9/1256\#BIBL

You can respond to this article at: http://ard.bmj.com/cgi/eletter-submit/67/9/1256

Receive free email alerts when new articles cite this article - sign up in the box at the top right corner of the article

Notes

To order reprints of this article go to:

http://journals.bmj.com/cgi/reprintform

To subscribe to Annals of the Rheumatic Diseases go to:

http://journals.bmj.com/subscriptions/ 


\title{
Association between leptin, body composition, sex and knee cartilage morphology in older adults: the Tasmanian older adult cohort (TASOAC) study
}

\author{
C Ding, ${ }^{1}$ V Parameswaran, ${ }^{2}$ F Cicuttini, ${ }^{3}$ J Burgess, ${ }^{2}$ G Zhai, ${ }^{1,4}$ S Quinn, ${ }^{1}$ G Jones ${ }^{1}$
}

${ }^{1}$ Menzies Research Institute, University of Tasmania, Hobart Tasmania, Australia; ${ }^{2}$ Diabetes and Endocrine Services, Royal Hobart Hospital, Hobart,

Tasmania, Australia;

${ }^{3}$ Department of Epidemiology and Preventive Medicine,

Monash University Medical School, Melbourne, Victoria, Australia; ${ }^{4}$ Twin Research \& Genetic Epidemiology Unit, King's College London School of Medicine, London, UK

Correspondence to:

Dr C Ding, Menzies Research Institute, Private Bag 23, Hobart, Tasmania 7000, Australia; changhai.ding@utas.edu.au

Accepted 20 December 2007

Published Online First

3 January 2008

\begin{abstract}
Objective: To describe the associations between leptin, body composition, sex and knee cartilage volume/defects in older adults.
\end{abstract}

Methods: A cross-sectional sample of 190 randomly selected subjects (mean 63 years, range 52-78, 48\% female) were studied. Knee cartilage volume and defects were determined using T1-weighted fat saturation MRI. Serum leptin levels were measured by radioimmunoassay. Fat and lean mass were measured by dual energy $x$ ray absorptiometry (DXA). Body mass index (BMI) was calculated.

Results: In multivariable analysis, serum levels of leptin were negatively associated with total cartilage volume $(\beta$ : $-541 \mathrm{~mm}^{3} / \log$ transformed unit, $95 \% \mathrm{Cl}-861$ to -221 ) but not with prevalent knee cartilage defects. BMI was negatively associated with cartilage volume after adjustment for total lean mass and positively with prevalent knee cartilage defects. However, the association between $\mathrm{BMI}$ and cartilage volume disappeared after adjustment for leptin while the association between BMl and cartilage defects remained unchanged. Lastly, sex differences in total cartilage volume decreased substantially after adjustment for leptin ( $R^{2}$ from $51 \%$ to $30 \%$ ).

Conclusions: This cross-sectional study suggests cartilage volume loss with obesity and female sex is related to leptin and, thus, is hormonally mediated in older adults. By contrast, obesity related knee focal cartilage defects may be more related to non-hormonal factors.

Osteoarthritis (OA) is a slowly progressive disease characterised by gradual loss of articular cartilage with a multifactorial cause. It is well established that obesity and female sex are risk factors for knee $\mathrm{OA} ;{ }^{1-3}$ however, the underlying mechanism remains obscure but may involve biomechanical processes ${ }^{4}$ or variations in sex hormones. ${ }^{2}$ Although there has been little evidence to show a metabolic link between obesity and knee $\mathrm{OA},{ }^{35}$ recent hypothetical arguments propose that leptin may represent a systemic factor linking obesity, sex and knee OA. ${ }^{67}$ Leptin, a $16 \mathrm{kDa}$ non-glycosylated protein encoded by the gene obese (ob), is a hormone secreted mainly by adipocytes ${ }^{8}$ as well as osteoblasts and chondrocytes. ${ }^{79}$ Leptin has been found in the synovial fluid of patients with $\mathrm{OA}^{9} 10$ and its concentration or mRNA expression in cartilage has been correlated with body mass index $(\mathrm{BMI})^{9}{ }^{10}$ and female sex. ${ }^{7}$ However, it is still not clear whether increased production of leptin is good or bad for cartilage health ${ }^{11}$ with recent evidence suggesting that leptin may act in a biphasic manner, ${ }^{12}$ ie, leptin physiologically may have a beneficial effect on cartilage synthesis, but an excess of leptin may lead to detrimental effects on cartilage..$^{10} 12$ There are no in vivo data to support this hypothesis to date.

Using MRI, we have demonstrated that BMI is positively associated with knee cartilage defects, ${ }^{13}$ and fat mass is negatively associated with knee cartilage volume. ${ }^{14}$ There are also large sex differences in knee cartilage volume in children ${ }^{15}$ and adults $^{2} 1617$ (males have 30-40\% higher cartilage volume than females). No studies have reported on whether leptin contributes to the BMI and sex related differences in cartilage morphology. The aim of this cross-sectional study, therefore, was to determine the associations between leptin and knee cartilage volume/defects in older adults, and explore the possible mediating roles of leptin in links between body mass, sex and cartilage volume/defects.

\section{MATERIALS AND METHODS \\ Subjects}

The study was carried out in southern Tasmania, Australia, from March until August 2002. Subjects aged between 50 and 79 years were selected randomly using computer generated random numbers from the electoral roll in southern Tasmania (population 229 000), a comprehensive population listing, with an equal number of men and women. Institutionalised persons were excluded. This study was conducted as part of the Tasmanian Older Adult Cohort (TASOAC) study, an ongoing, prospective, population-based study in 1100 subjects aimed at identifying the environmental, genetic and biochemical factors associated with the development and progression of osteoarthritis and osteoporosis (the overall response rate was $57 \%$ ). We selected the first 190 subjects to perform serum leptin measurement. The study was approved by the Southern Tasmanian Health and Medical Human Research Ethics Committee, and written informed consent was obtained from all participants. Self-report of smoking status and disease status such as rheumatoid arthritis (RA), asthma, cardiovascular disease and diabetes were recorded by questionnaire.

\section{Anthropometrics}

Height was measured to the nearest $0.1 \mathrm{~cm}$ (with shoes, socks and headgear removed) using a stadiometer. Weight was measured to the nearest $0.1 \mathrm{~kg}$ (with shoes, socks and bulky clothing removed) using a single pair of electronic scales (Seca Delta Model 707, Bradford, Massachusetts, 
Table 1 Characteristics of participants

\begin{tabular}{|c|c|c|c|}
\hline & $\begin{array}{l}\text { Leptin < } \\
\text { median }(n=96)\end{array}$ & $\begin{array}{l}\text { Leptin } \geqslant \\
\text { median ( } n=94)\end{array}$ & p Values \\
\hline Age, years & $62.7(7.2)$ & $62.6(6.9)$ & 0.916 \\
\hline Female sex, \% & 17 & 79 & $<0.001$ \\
\hline Body mass index, $\mathrm{kg} / \mathrm{m}^{2}$ & $26.0(3.4)$ & $29.1(4.7)$ & $<0.001$ \\
\hline Total lean mass, kg & $57.5(9.4)$ & $47.8(10.0)$ & $<0.001$ \\
\hline Total fat mass, \% & $28.2(4.7)$ & $39.2(5.9)$ & $<0.001$ \\
\hline Trunk fat mass, $\%$ & $27.8(5.3)$ & $38.3(5.7)$ & $<0.001$ \\
\hline Current smokers, \% & 17 & 15 & 0.889 \\
\hline Rheumatoid arthritis, \% & 8 & 14 & 0.227 \\
\hline Asthma, \% & 6 & 24 & 0.001 \\
\hline Cardiovascular diseases, \% & 5 & 3 & 0.489 \\
\hline Diabetes, \% & 3 & 8 & 0.182 \\
\hline Knee pain, \% & 40 & 53 & 0.071 \\
\hline Medial joint space narrowing, \% & 48 & 56 & 0.266 \\
\hline Lateral joint space narrowing, \% & 16 & 15 & 0.923 \\
\hline Medial tibiofemoral osteophytes, \% & 7 & 7 & 0.952 \\
\hline Lateral tibiofemoral osteophytes, \% & 3 & 3 & 0.967 \\
\hline Prevalent medial cartilage defects, $\%$ & 25 & 22 & 0.543 \\
\hline Prevalent lateral cartilage defects, $\%$ & 19 & 17 & 0.622 \\
\hline Prevalent patellar cartilage defects, $\%$ & 34 & 44 & 0.194 \\
\hline Prevalent total cartilage defects, \% & 46 & 54 & 0.246 \\
\hline Total cartilage defects, $0-20$ & $5.7(2.4)$ & $6.0(1.9)$ & 0.506 \\
\hline Medial tibial cartilage volume, ml & $3.0(0.3)$ & $2.4(0.5)$ & $<0.001$ \\
\hline Lateral tibial cartilage volume, $\mathrm{ml}$ & $3.4(0.7)$ & $2.6(0.5)$ & $<0.001$ \\
\hline Patellar cartilage volume, ml & $3.9(0.9)$ & $3.1(0.8)$ & $<0.001$ \\
\hline Total cartilage volume, $\mathrm{ml}$ & $10.2(1.6)$ & $8.0(1.5)$ & $<0.001$ \\
\hline
\end{tabular}

Data shown are mean (SD) or median (interquartile range), except for percentages. Median value of leptin: $8.7 \mu \mathrm{g} /$ /itre.

USA) that were calibrated using a known weight at the beginning of each clinic. The body mass index (BMI; weight $(\mathrm{kg}) /$ height $\left.\left(\mathrm{m}^{2}\right)\right)$ was calculated. Fat mass and lean mass $(\mathrm{kg})$ were measured by a Hologic dual energy $x$ ray absorptiometry (DXA) scanner (Hologic Corp., Waltham, Massachusetts, USA). Percentage total body or trunk fat mass is the ratio of total body or trunk fat mass divided by total body or trunk mass (ie, the sum of fat mass, lean mass and bone mass). $x$ Ray and knee pain assessment

A standing anteroposterior semiflexed view of the right knee with $15^{\circ}$ of fixed knee flexion was performed in all subjects at baseline and scored individually for osteophytes and joint space narrowing on a scale of $0-3 \quad(0=$ normal and $3=$ severe $)$ according to the Osteoarthritis Research Society International (OARSI) atlas as previously described. ${ }^{18}$ The presence of radiographic OA (ROA) was defined as any score of $\geqslant 1$.

Knee pain (on flat surface, going up/down stairs, at night, sitting/lying and standing upright) was assessed by selfadministered questionnaire using the Western Ontario McMaster Osteoarthritis Index (WOMAC) with a 10-point scale from 0 (no pain, stiffness or no function problems) to 9 (most severe pain, stiffness or severe function problems). ${ }^{19}$ Each score of knee pain was summed to create a total pain (0-45) score. Prevalent knee pain was defined as a total score of $\geqslant 1$.

\section{Knee cartilage volume measurement}

Knee cartilage volume was determined by means of image processing on an independent workstation as previously described. ${ }^{15} 20$ The volumes of individual cartilage plates (medial tibial, lateral tibial and patella) were isolated from the total volume by manually drawing disarticulation contours around the cartilage boundaries on a section by section basis. These data were then resampled by means of bilinear and cubic interpolation (area of 312 and $312 \mu \mathrm{m}$ and $1.5 \mathrm{~mm}$ thickness, continuous sections) for the final $3 \mathrm{D}$ rendering. The coefficients of variation (CVs) for cartilage volume measures were $2.1-2.6 \%$. The sum of medial tibial, lateral tibial and patellar cartilage volume was regarded as "total" cartilage volume.

Cartilage defects ( $0-4$ scale) were graded at medial tibial, medial femoral, lateral tibial, lateral femoral and patellar sites as follows: ${ }^{13}{ }^{21}$ grade 0 , normal cartilage; grade 1 , focal blistering and intracartilaginous low-signal intensity area with an intact surface and bottom; grade 2, irregularities on the surface or bottom and loss of thickness of less than $50 \%$; grade 3 , deep ulceration with loss of thickness of more than $50 \%$; grade 4 , fullthickness chondral wear with exposure of subchondral bone. A cartilage defect also had to be present in at least two consecutive slices. A prevalent cartilage defect was defined as
Figure 1 Scatter-plot for the association between serum levels of leptin and total knee cartilage volume. Log transformed leptin was significantly associated with knee cartilage volume $(r=-0.52$, $\mathrm{p}<0.001$ ) in unadjusted analysis.

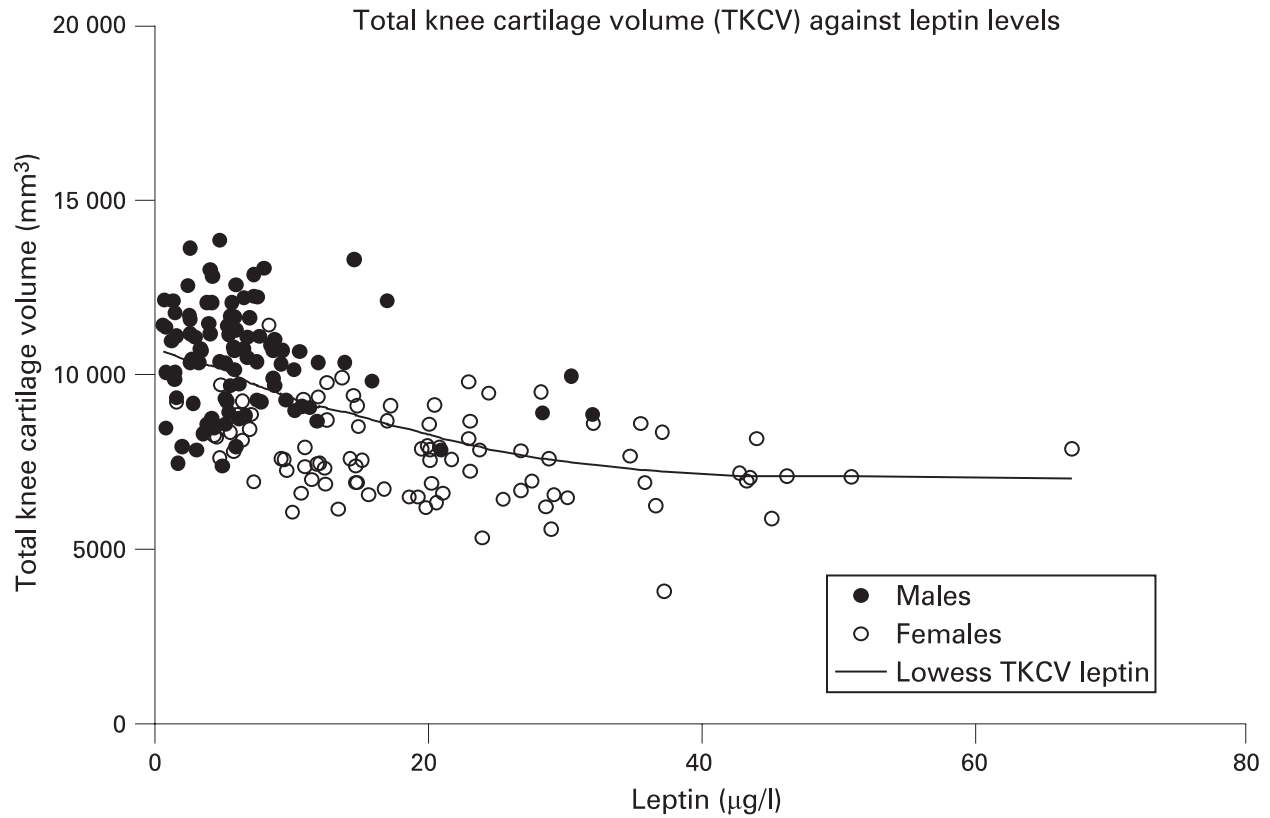


Table 2 Associations between leptin, knee cartilage volume and cartilage defects

\begin{tabular}{|c|c|c|}
\hline & Univariable & Multivariable* \\
\hline \multicolumn{3}{|c|}{ Cartilage volume, $\beta$ (95\% Cl): } \\
\hline Total & $-1028(-1270$ to -787$)$ & $-541(-861$ to -221$)$ \\
\hline Medial tibial & $-298(-375$ to -221$)$ & $-183(-300$ to -66$)$ \\
\hline Lateral tibial & $-350(-441$ to -259$)$ & $-161(-294$ to -28$)$ \\
\hline Patellar & $-380(-501$ to -260$)$ & $-183(-340$ to -26$)$ \\
\hline \multicolumn{3}{|c|}{ Cartilage defects, odds ratio $(95 \% \mathrm{Cl})$ : } \\
\hline Total & $1.13(0.84$ to 1.51$)$ & 0.89 (0.52 to 1.52$)$ \\
\hline Medial tibiofemoral & 0.91 (0.65 to 1.28$)$ & $0.60(0.32$ to 1.13$)$ \\
\hline Lateral tibiofemoral & $1.01(0.69$ to 1.16$)$ & $1.09(0.54$ to 2.23$)$ \\
\hline Patellar & $1.15(0.85$ to 1.55$)$ & $0.73(0.43$ to 1.23$)$ \\
\hline
\end{tabular}

Dependent variable: cartilage volume, $\mathrm{mm}^{3}$ or prevalent castilage defects. Independent variable: leptin, per unit. Data in bold denotes a statistically significant result.

${ }^{*}$ Adjusted for sex, age, body mass index (BMI), smoking, knee joint space narrowing, osteophytes, rheumatoid arthritis (RA), asthma, cardiovascular diseases and cartilage defects if cartilage volume or cartilage volume if cartilage defects.

a cartilage defect score of $\geqslant 2$ at any site within that compartment. Intraobserver reliability (expressed as intraclass correlation coefficient (ICC)) was 0.89-0.94 and interobserver reliability was $0.85-0.93 .^{21}$

Tibial bone area at the medial and lateral compartments was determined as previously described. ${ }^{18}$

\section{Serum leptin measurement}

Serum was separated and aliquotted into plastic storage tubes. Aliquots were stored at $-80^{\circ} \mathrm{C}$ till analysis. The levels of leptin were measured by radioimmunoassay (LINCO Research; now part of Millipore, Missouri, USA). Samples with undetectable leptin concentration were assigned a value corresponding to the lower limit of detection of the assay $(0.5 \mu \mathrm{g} /$ litre $)$. The intra and inter assay CVs were $4.6 \%$ and $5 \%$ respectively in our hands

\section{Data analysis}

Student t or $\chi^{2}$ tests (where appropriate) were used to compare means. A scatter plot and locally weighted scatterplot smoothing (lowess) plot were used to depict the association between unadjusted leptin levels and total knee cartilage volume. The lowess command models the data locally using a cubic weighted least squares regression, with weights that give more importance to local data points. Leptin was not normally distributed, hence was log transformed for analyses. Univariable and multivariable linear regression analyses were used to examine the associations between knee cartilage volume and log transformed leptin before and after adjustment age, sex, BMI, smoking status, ROA, bone size, other disease status (RA, cardiovascular disease, asthma and diabetes). The associations between knee cartilage volume and BMI (or body fat mass) were also investigated by linear regression after adjustment for leptin. Similarly, univariable and multivariable logistic regression analyses were used to examine the associations between prevalent knee cartilage defects and log transformed leptin/ BMI/body fat mass. Standard diagnostic checks of model fit and residuals were routinely made, and data points with large residuals and/or high influence were investigated for data errors. A $p$ value $<0.05$ (two-tailed) or a $95 \%$ confidence interval not including the null point were regarded as statistically significant. All statistical analyses were performed on SPSS V.12.0 for Windows (SPSS, Chicago, Illinois, USA) or Stata statistical software, V.9.2 (Stata Corp., College Station, Texas, USA).

\section{RESULTS}

A total of 190 subjects (48\% female) aged between 52 and 78 (mean 63 years) participated in the present study. There were no significant differences in demographic factors and knee cartilage volume between the current cohort and the subjects who did not have serum markers measured (mean (SD) age: 62.6 (7.1) vs 62.3 (7.5) years, $p=0.57$; female sex: $48 \%$ vs $50 \%$, $\mathrm{p}=0.62$; BMI: 27.5 (4.4) vs 27.7 (4.5) kg/m², $\mathrm{p}=0.68$; and total cartilage volume: 9.1 (1.9) vs $9.2(1.8) \mathrm{ml}, \mathrm{p}=0.42)$. This is a mixed population with $58 \%$ subjects having radiographic changes (joint space narrowing or osteophytes score of $\geqslant 1$ ) in the right knee and $46 \%$ having knee pain. Characteristics of the subjects are presented in table 1 . Subjects with higher and lower levels of leptin (split at the median of $8.7 \mu \mathrm{g} /$ litre) were similar in terms of age, smoking status, knee pain, radiographic joint space narrowing, osteophytes, prevalent cartilage defects and

Table 3 Associations between BMI, knee cartilage volume and cartilage defects: mediating roles of leptin

\begin{tabular}{|c|c|c|c|c|}
\hline & Univariable & Multivariable* & Multivariable $\dagger$ & Multivariable: \\
\hline \multicolumn{5}{|l|}{ Cartilage volume, $\beta(95 \% \mathrm{Cl})$ : } \\
\hline Total cartilage & $-48(-112$ to 16$)$ & $-99(-144$ to -53$)$ & $-51(-98$ to -4$)$ & $-2(-61$ to 56$)$ \\
\hline Medial tibial cartilage & $-14(-34$ to 6$)$ & $-27(-43$ to -11$)$ & $-14(-32$ to 3$)$ & $+3(-19$ to 24$)$ \\
\hline Patellar cartilage & $-10(-40$ to 20$)$ & $-31(-55$ to -8$)$ & $-21(-47$ to 4$)$ & $-10(-42$ to 23$)$ \\
\hline \multicolumn{5}{|c|}{ Cartilage defects, odds ratio $(95 \% \mathrm{CI})$ : } \\
\hline Total cartilage & $1.06(0.99$ to 1.13$)$ & - & 1.10 (1.01 to 1.19$)$ & 1.12 (1.01 to 1.24$)$ \\
\hline Patellar cartilage & $1.08(1.01$ to 1.16$)$ & - & 1.14 (1.06 to 1.24$)$ & 1.19 (1.07 to 1.32$)$ \\
\hline
\end{tabular}

Dependent variable: cartilage volume, $\mathrm{mm}^{3}$ or prevalent cartilage defects. Independent variable: body mass index (BMI), $\mathrm{kg} / \mathrm{m}^{2}$. Data in bold denotes a statistically significant result. ${ }^{*}$ Adjusted for total lean mass; ffurther adjusted for sex, age, smoking, knee joint space narrowing, osteophytes, rheumatoid arthritis (RA), asthma, diabetes and cardiovascular diseases; ffurther adjusted for leptin. 
Table 4 Associations between body composition and knee cartilage volume: mediating roles of leptin

\begin{tabular}{|c|c|c|c|}
\hline & Univariable & Multivariable* & Multivariable $\dagger$ \\
\hline \multicolumn{4}{|c|}{ Total cartilage, $\beta(95 \% \mathrm{Cl})$ : } \\
\hline Trunk fat, \% & $-147(-177$ to -116$)$ & $-98(-154$ to -42$)$ & $-75(-134$ to -16$)$ \\
\hline Total fat, $\%$ & $-173(-199$ to -146$)$ & $-111(-185$ to -38$)$ & $-80(-156$ to -5$)$ \\
\hline \multicolumn{4}{|c|}{$\begin{array}{l}\text { Medial tibial cartilage, } \\
\beta(95 \% \mathrm{CI}) \text { : }\end{array}$} \\
\hline Trunk fat, \% & $-41(-51$ to -31$)$ & $-31(-50$ to -11$)$ & $-22(-43$ to -2$)$ \\
\hline Total fat, $\%$ & $-47(-56$ to -38$)$ & $-31(-57$ to -5$)$ & $-20(-47$ to 7$)$ \\
\hline \multicolumn{4}{|c|}{$\begin{array}{l}\text { Lateral tibial cartilage, } \\
\beta(95 \% \mathrm{CI})\end{array}$} \\
\hline Trunk fat, \% & $-51(-63$ to -40$)$ & $-32(-54$ to -10$)$ & $-27(-51$ to -3$)$ \\
\hline Total fat, \% & $-59(-69$ to -49$)$ & $-42(-72$ to -13$)$ & $-35(-66$ to -5$)$ \\
\hline \multicolumn{4}{|c|}{ Patellar cartilage, $\beta(95 \% \mathrm{CI})$ : } \\
\hline Trunk fat, \% & $-54(-70$ to -39$)$ & $-37(-63$ to -11$)$ & $-30(-58$ to -2$)$ \\
\hline Total fat, $\%$ & $-66(-80$ to -52$)$ & $-46(-81$ to -10$)$ & $-35(-72$ to 2$)$ \\
\hline
\end{tabular}

total cartilage defect scores. However, subjects with higher levels of leptin had a higher proportion of female sex, greater BMI, less lean mass and greater \% fat mass, higher prevalence of asthma and lower cartilage volume.

Leptin levels were significantly associated with knee cartilage volume before (fig 1, table 2) and after adjustment for sex, age, BMI, smoking, ROA, cartilage defects and other disease status. The associations remained largely unchanged after further adjustment for tibial bone size. By contrast, no significant associations were determined between prevalent cartilage defects and leptin in univariable and multivariable analyses (table 2).

BMI was significantly associated with leptin before and after adjustment for covariates (partial $r=0.62, p<0.001$ ) in this sample. In table 3 , although most associations between BMI and knee cartilage volume in the univariable analyses were not significant, BMI was significantly negatively associated with knee cartilage volume after adjustment for lean mass (as total lean mass was positively associated with total cartilage volume $(p<0.001))$. The associations between BMI, and total and lateral tibial cartilage volume remained significant after further adjustment for other covariates; however, these associations became non-significant after further adjustment for leptin (table 3). In addition, BMI was significantly positively associated with prevalent total and patellar cartilage defects but, in contrast, these associations did not change after further adjustment for leptin (table 3 ).

Percentage total fat and trunk fat mass was also positively associated with leptin in multivariable analyses (partial $r=0.31$ and $r=0.36$, respectively, both $p<0.001$ ). In table 4 , percentage total fat mass and trunk fat mass were negatively associated with knee cartilage volume. These associations decreased by $25-$ $30 \%$ after adjustment for leptin (table 4). By contrast, there were no significant association between fat mass and knee cartilage defects, and these associations were not altered by further adjustment for leptin (data not shown).

There were large sex differences in leptin levels (women vs men: $+12 \mu \mathrm{g} /$ litre, $\left.\mathrm{R}^{2}=43 \%, \mathrm{p}<0.001\right)$ and total knee cartilage volume $\left(R^{2}=51 \%\right.$, table 5$)$. The sex difference in total knee cartilage volume decreased substantially in magnitude but remained significant after adjustment for leptin $\left(\mathrm{R}^{2}=30 \%\right)$ (table 5). The association between sex and prevalent total cartilage defects was not significant in this sample $(\mathrm{OR}=1.20$, $p=0.60)$, and was not influenced by adjustment for leptin.

No significant association was present between leptin and ROA in adjusted analysis (data not shown). Subjects with higher leptin levels had a trend to higher prevalence of knee pain and medial joint space narrowing (JSN) but these were not significant (table 1). The association between leptin and medial JSN $(O R=1.10, p=0.65)$ remained unchanged after adjustment for knee pain $(O R=1.07, p=0.76)$. The strength of the associations and the evidence of mediation by leptin did not differ when the analysis were performed separately in men and women (data not shown). No significant interaction was present between sex and leptin $(p=0.92)$ or radiographic $\mathrm{OA}$ and leptin $(\mathrm{p}=0.64)$ for total cartilage volume, so all data were combined for analyses. Results remained largely unchanged when subjects with RA, cardiovascular diseases, diabetes, or asthma were excluded for analyses (data not shown).

Table 5 Associations between sex and knee cartilage volume: mediating roles of leptin

\begin{tabular}{|c|c|c|c|}
\hline & Univariable & Multivariable* & Multivariable $\dagger$ \\
\hline & $\beta(95 \% \mathrm{Cl}) / \mathrm{R}^{2}$ & $\beta(95 \% \mathrm{CI}) /$ partial R ${ }^{2}$ & $\beta(95 \% \mathrm{CI}) /$ partial R ${ }^{2}$ \\
\hline Total cartilage & $-2764(-3154$ to -2374$) / R^{2}: 51 \%$ & $-2669(-3076$ to -2261$) / R^{2}: 51 \%$ & $-2174(-2694$ to -1654$) / R^{2}: 30 \%$ \\
\hline Lateral tibial cartilage & $-909(-1066$ to -752$) / R^{2}: 41 \%$ & $-861(-1025$ to -697$) / R^{2}: 40 \%$ & $-699(-910$ to -489$) / R^{2}: 21 \%$ \\
\hline Patellar cartilage & $-1092(-1300$ to -885$) / R^{2}: 36 \%$ & $-1046(-1265$ to -828$) / R^{2}: 34 \%$ & $-909(-1196$ to -621$) / R^{2}: 18 \%$ \\
\hline
\end{tabular}

Dependent variable: cartilage volume, $\mathrm{mm}^{3}$. Independent variable: $\operatorname{sex}(\mathrm{men}=0$, women $=1)$. All results were statistically significant.

*Adjusted for age, body mss index (BMI), smoking, knee joint space narrowing, osteophytes, rheumatoid arthritis (RA), asthma, diabetes and cardiovascular diseases; †further adjusted for leptin. 


\section{DISCUSSION}

This study reports a consistent negative association between serum leptin levels and knee cartilage volume (both total and in all compartments) in older adults with or without radiographic $\mathrm{OA}$. This was independent of BMI and sex. Conversely, the associations between BMI, body fat mass, sex and knee cartilage volume were not independent of serum leptin levels. Lastly, the association between BMI and prevalent knee cartilage defects was not related to leptin.

Assessment of cartilage volume and cartilage defects by MRI, with fat-suppressed gradient echo sequences, and appropriate image analysis techniques, has high accuracy and adequate precision for cross-sectional and longitudinal studies in healthy subjects and patients with $O A .{ }^{22}{ }^{23}$ Cartilage defects and volume are both important in OA. Cartilage defects seem to represent an early stage of cartilage damage and predict cartilage volume loss in a number of studies. ${ }^{23}$ Independent to the association with cartilage defects, cartilage volume is also impacted on by other structural and, based on this study, hormonal factors. We found that higher serum leptin levels were significantly associated with lower knee cartilage volume at all three sites. This is in line with a recent in vitro study that suggested that leptin and its receptor (ob-Rb) mRNA was expressed at significantly higher levels in osteoarthritic compared to normal cartilage, and leptin had a detrimental effect on chondrocyte proliferation and induced matrix metalloproteinase (MMP) 9 and 13 protein expression. ${ }^{10}$ Although studies suggest that leptin levels are higher in synovial fluid than in serum, ${ }^{10}{ }^{24}$ our data clearly demonstrates that serum leptin levels are associated with reduced knee cartilage volume in one knee, suggesting a systemic hormonal influence on cartilage volume. Whether this reflects a systemic effect on other joints with hyaline cartilage is unknown, but is deserving of further study. The associations between leptin and knee cartilage volume remained significant after adjustment for BMI, suggesting its effects on knee cartilage are independent of BMI. Of note, we found that medial joint space narrowing scores were worse in subjects with high leptin levels (>median value) but this was not significant, most likely indicating lower sensitivity for radiographs compared with MRI. A more sensitive protocol for the radiography, eg, the Lyon schuss view, may show such a difference or it may be that all radiographs lack sensitivity.

Consistent with our previous report, ${ }^{1}$ BMI was not significantly associated with knee cartilage volume in unadjusted analysis. However, BMI was significantly negatively associated with knee cartilage volume when the protective effect of lean mass was taken into account (by adjustment for lean mass). Similarly, fat mass was negatively associated with knee cartilage volume, which is also consistent with a recent report. ${ }^{14}$ The associations of BMI and fat mass with cartilage volume were dependent on leptin, suggesting that at least some of the association between obesity and cartilage volume is hormonally linked rather than purely mechanical. By contrast, we did not find significant associations between serum leptin and knee focal cartilage defects even though cartilage defects were significantly associated with loss of cartilage volume. ${ }^{23}$ Although obesity was associated with cartilage defects as we have previously reported, ${ }^{1}$ adjustment for leptin did little to this suggesting cartilage splitting or focal defects are more susceptible to mechanical loading ${ }^{25}$ than hormonal effects.

Males have consistently higher knee cartilage volumes than females, ${ }^{15-1726}$ as seen in the findings from this study. This is predominantly due to greater body size. Other factors may include sex hormones and growth factors. ${ }^{16}$ Variation in leptin may be another contributor. Indeed, we found that older females had significantly greater serum leptin levels than older males, and sex differences in knee cartilage volume decreased substantially in magnitude after adjustment for leptin providing support for this hypothesis.

Our study has several potential limitations. First, the sample size was relatively small, which may miss moderate associations; however, significant associations between leptin and knee cartilage volume were detected using this sample size. Second, the response rate at baseline was $57 \%$, which may leave the possibility open for selection bias although there were no differences between those reported in this study and the rest of the cohort. Third, this randomly selected sample contained subjects with other diseases. However, the results were largely unchanged when the analyses were adjusted for disease status or these subjects were excluded. Fourth, we used tibial cartilage as the measure of joint cartilage at the tibiofemoral joint rather that femoral cartilage, and it is possible that associations with femoral cartilage are different to those with tibial cartilage. ${ }^{27}$ However, we have previously shown a strong correlation between the tibial and femoral cartilage in the medial and lateral tibiofemoral compartments. ${ }^{28}$ It would be worthwhile to examine associations at other sites, eg, hip and spinal discs. Fifth, measurement error may influence results. However, all measures were highly reproducible suggesting this is unlikely. Lastly, due to cross-sectional nature, this study cannot exclude the possible confounding effect on cartilage volume measurements of hypertrophic repair or swelling in OA cartilage. The cause effect relationship between obesity, sex, leptin and cartilage needs to be elucidated by longitudinal studies.

In conclusion, this cross-sectional study suggests that cartilage volume loss with obesity and female sex is related to leptin and, thus, is hormonally mediated in older adults. By contrast, obesity related knee focal cartilage defects may be more related to non-hormonal factors.

Acknowledgements: Special thanks go to the subjects who made this study possible. The role of Catrina Boon and Pip Boon in collecting the data is gratefully acknowledged. We would also like to thank Drs V Srikanth and H Cooley for radiographic assessment.

Funding: Funding was received from the National Health and Medical Research Council of Australia (302204), the Tasmanian Community Fund (D0015018), the Arthritis Foundation of Australia (MRI06161) and the University of Tasmania GrantInstitutional Research Scheme (D0015019).

\section{Competing interests: None.}

Ethics approval: The study was approved by the Southern Tasmanian Health and Medical Human Research Ethics Committee, and written informed consent was obtained from all participants.

\section{REFERENCES}

1. Ding C, Cicuttini F, Scott F, Cooley H, Jones G. Knee structural alteration and BMI: a cross-sectional study. Obes Res 2005;13:350-61.

2. Ding C, Cicuttini F, Blizzard L, Scott F, Jones G. A longitudinal study of the effect of sex and age on rate of change in knee cartilage volume in adults. Rheumatology (Oxford) 2007;:46:273-9.

3. Sharma L, Chang A. Overweight: advancing our understanding of its impact on the knee and the hip. Ann Rheum Dis 2007:66:141-2.

4. Messier SP, Gutekunst DJ, Davis C, DeVita P. Weight loss reduces knee-joint loads in overweight and obese older adults with knee osteoarthritis. Arthritis Rheum 2005;52:2026-32.

5. Aspden RM, Scheven BA, Hutchison JD. Osteoarthritis as a systemic disorder including stromal cell differentiation and lipid metabolism. Lancet 2001;357:1118-20.

6. Gualillo $\mathbf{0}$. Further evidence for leptin involvement in cartilage homeostases. Osteoarthritis Cartilage 2007;15:857-60.

7. Teichtahl AJ, Wluka AE, Proietto J, Cicuttini FM. Obesity and the female sex, risk factors for knee osteoarthritis that may be attributable to systemic or local leptin biosynthesis and its cellular effects. Med Hypotheses 2005;65:312-5.

8. Zhang Y, Proenca R, Maffei M, Barone M, Leopold L, Friedman JM. Positional cloning of the mouse obese gene and its human homologue. Nature 1994;372:425-32. 
9. Dumond H, Presle N, Terlain B, Mainard D, Loeuille D, Netter P, et al. Evidence for a key role of leptin in osteoarthritis. Arthritis Rheum 2003;48:3118-29.

10. Simopoulou T, Malizos KN, lliopoulos D, Stefanou N, Papatheodorou L, loannou M, et al. Differential expression of leptin and leptin's receptor isoform (Ob-Rb) mRNA between advanced and minimally affected osteoarthritic cartilage; effect on cartilage metabolism. Osteoarthritis Cartilage 2007;15:872-83.

11. Loeser RF. Systemic and local regulation of articular cartilage metabolism: where does leptin fit in the puzzle? Arthritis Rheum 2003:48:3009-12.

12. Pottie $\mathbf{P}$, Presle N, Terlain B, Netter P, Mainard D, Berenbaum F. Obesity and osteoarthritis: more complex than predicted! Ann Rheum Dis 2006;65:1403-5.

13. Ding C, Cicuttini F, Scott F, Cooley H, Boon C, Jones G. Natural history of knee cartilage defects and factors affecting change. Arch Intern Med 2006;166:651-8.

14. Wang Y, Wluka AE, English DR, Teichtahl AJ, Giles GG, O'Sullivan R, et al. Body composition and knee cartilage properties in healthy, community-based adults. Ann Rheum Dis 2007:66:1244-8.

15. Jones G, Glisson M, Hynes K, Cicuttini F. Sex and site differences in cartilage development: a possible explanation for variations in knee osteoarthritis in later life. Arthritis Rheum 2000;43:2543-9.

16. Ding C, Cicuttini F, Scott F, Glisson M, Jones G. Sex differences in knee cartilage volume in adults: role of body and bone size, age and physical activity. Rheumatology (Oxford) 2003:42:1317-23.

17. Faber SC, Eckstein F, Lukasz S, Muhlbauer R, Hohe J, Englmeier KH, et al. Gender differences in knee joint cartilage thickness, volume and articular surface areas: assessment with quantitative three-dimensional MR imaging. Skeletal Radiol 2001;30:144-50.

18. Jones G, Ding C, Scott F, Glisson M, Cicuttini F. Early radiographic osteoarthritis is associated with substantial changes in cartilage volume and tibial bone surface area in both males and females. Osteoarthritis Cartilage 2004;12:169-74.

19. Bellamy N, Buchanan WW, Goldsmith CH, Campbell J, Stitt LW. Validation study of WOMAC: a health status instrument for measuring clinically important patient relevant outcomes to antirheumatic drug therapy in patients with osteoarthritis of the hip or knee. J Rheumatol 1988;15:1833-40.

20. Ding C, Cicuttini F, Blizzard L, Jones G. Smoking interacts with family history with regard to knee cartilage loss and cartilage defect development. Arthritis Rheum 2007:56:1521-8.

21. Ding C, Garnero P, Cicuttini F, Scott F, Cooley H, Jones G. Knee cartilage defects: association with early radiographic osteoarthritis, decreased cartilage volume, increased joint surface area and type II collagen breakdown. Osteoarthritis Cartilage 2005; 13:198-205

22. Eckstein F, Cicuttini F, Raynauld JP, Waterton JC, Peterfy C. Magnetic resonance imaging (MRI) of articular cartilage in knee osteoarthritis (OA): morphological assessment. Osteoarthritis Cartilage 2006;14(Suppl 1):46-75.

23. Ding C, Cicuttini F, Jones G. Tibial subchondral bone size and knee cartilage defects: relevance to knee osteoarthritis. Osteoarthritis Cartilage 2007;15:479-86.

24. Presle N, Pottie P, Dumond H, Guillaume C, Lapicque F, Pallu S, et al. Differential distribution of adipokines between serum and synovial fluid in patients with osteoarthritis. Contribution of joint tissues to their articular production. Osteoarthritis Cartilage 2006:14:690-5.

25. Janakiramanan $\mathbf{N}$, Teichtahl AJ, Wluka AE, Ding C, Jones G, Davis SR, et al. Static knee alignment is associated with the risk of unicompartmental knee cartilage defects. J Orthop Res 2008:26:225-30.

26. Cicuttini F, Forbes A, Morris K, Darling S, Bailey M, Stuckey S. Gender differences in knee cartilage volume as measured by magnetic resonance imaging. Osteoarthritis Cartilage 1999;7:265-71

27. Ding C, Martel-Pelletier J, Pelletier JP, Abram F, Raynauld JP, Cicuttini F, et al. Twoyear prospective longitudinal study exploring the factors associated with change in femoral cartilage volume in a cohort largely without knee radiographic osteoarthritis. Osteoarthritis Cartilage 2008;16:443-9.

28. Cicuttini FM, Wluka AE, Stuckey SL. Tibial and femoral cartilage changes in knee osteoarthritis. Ann Rheum Dis 2001;60:977-80.

\section{BMJ Masterclasses}

BMJ Masterclasses are educational meetings designed specifically to meet the learning needs of doctors. They help doctors keep up to date with the latest evidence and recent guidelines in major clinical areas, enabling them to use the latest evidence to make better decisions. The latest evidence, recent guidelines and best practice are delivered in an interactive and informative manner by leading experts. The speakers are specifically chosen as highly-skilled communicators who can authoritatively enthuse the audience and interpret the latest research and guidelines into practical tips for busy doctors. BMJ Masterclasses have proved a huge hit with clinicians, with many saying they have influenced their clinical practice.

http://masterclasses.bmj.com/

\section{$\mathrm{BMJ}$}

masterclasses 